\title{
Efficient Silicon-on-Insulator Fiber Coupler Fabricated Using 248-nm-Deep UV Lithography
}

\author{
Gunther Roelkens, Student Member, IEEE, Pieter Dumon, Student Member, IEEE,
} Wim Bogaerts, Student Member, IEEE, Dries Van Thourhout, Member, IEEE, and Roel Baets, Senior Member, IEEE

\begin{abstract}
We present a silicon-on-insulator (SOI) waveguide to fiber coupler fabricated using 248-nm-deep ultraviolet lithography. The loss of the taper structure is around $1 \mathrm{~dB}$ while the coupling loss from a lensed fiber into a 590-nm-wide SOI waveguide was measured to be $1.9 \mathrm{~dB}$.
\end{abstract}

Index Terms - Integrated optics, silicon-on-insulator technology.

\section{INTRODUCTION}

I N THE last years, silicon-on-insulator (SOI) is emerging as a very promising platform for photonic integration. Due to the high omni-directional index contrast very compact wavelength scale components can be fabricated. Moreover, one can use the standard complementary metal-oxide-semiconductor (CMOS) technology to mass manufacture these optical devices [1]. However, the viability of integrated optical components depends just as much on the capability to provide them with manufacturable low-loss interfaces to an optical fiber. In this letter, we demonstrate that CMOS technology can also play a key role for these interfaces.

\section{Fiber to WAVEGUide COUPLING}

The coupling of light from an optical fiber into an SOI waveguide is made difficult by the small overlap of the SOI waveguide mode and the fiber waveguide mode. Several approaches are proposed in the literature to tackle this problem. Grating couplers are good candidates due to the lack of the need for cleaved facets.

However they intrinsically suffer from a compromise between efficiency and optical bandwidth, making them unsuitable in some applications [2]. Another approach presented in the literature is to use a spot size converter to transform the SOI waveguide mode to a polymer waveguide mode which matches a lensed fiber mode, as shown in Fig. 1 [3], [4]. The optical bandwidth is typically very large $(>100 \mathrm{~nm})$ and efficiencies are high $(<1-\mathrm{dB}$ loss $)$. To obtain this high efficiency, the SOI taper needs to taper accurately to widths below $100 \mathrm{~nm}$ for

Manuscript received July 11, 2005; revised August 11, 2005. This work was supported in part by the European Union under the IST project PICMOS (FP62002-IST-1-002131) and by the IST Network of Excellence ePIXnet. The work of G. Roelkens and W. Bogaerts was supported by the Fund for Scientific Research (FWO), and by the IAP-Photon Network. The work of P. Dumon was supported by a scholarship from the Institute for the Promotion of Innovation through Science and Technology in Flanders (IWT Vlaanderen).

The authors are with the Department of Information Technology (INTEC), Ghent University, B-9000 Gent, Belgium (e-mail: Gunther. Roelkens@intec.ugent.be; Pieter.Dumon@intec.ugent.be; Wim.Bogaerts@ intec.ugent.be; driesvt@intec.ugent.be; Roel.Baets@intec.ugent.be).

Digital Object Identifier 10.1109/LPT.2005.859132

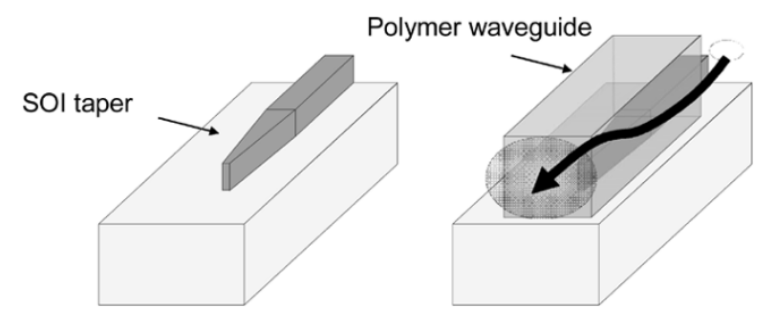

Fig. 1. SOI waveguide spot size converter for efficient coupling to a lensed fiber.

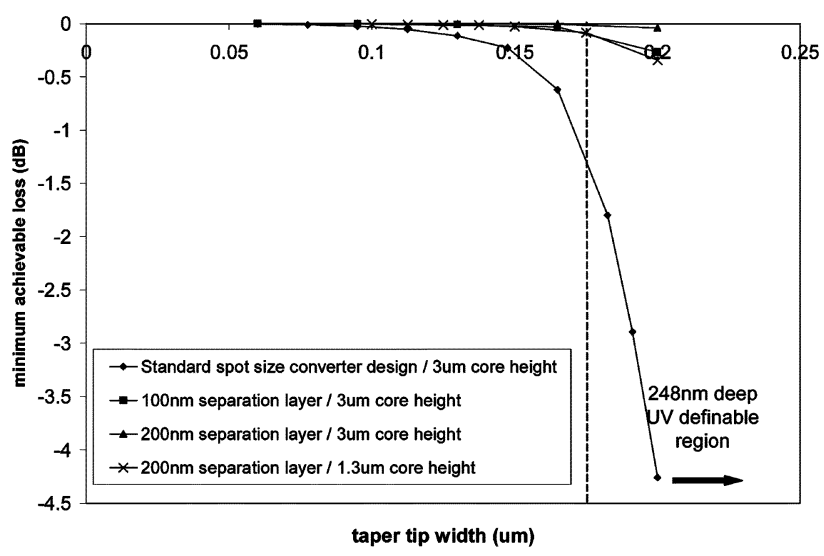

Fig. 2. Minimum achievable loss of the spot size converter as a function of SOI taper tip width. Simulations are for TE polarization and a wavelength of $1.55 \mu \mathrm{m}$.

high efficiency due to the strong optical confinement in the SOI waveguide. While this is not a problem for an e-beam lithography system in a research environment, this is much more difficult to achieve for the standard industrial CMOS 248-nm-deep ultraviolet (UV) lithography machines. In Fig. 2, we present the minimum achievable loss of the standard spot size converter design as a function of the taper width at $1.55-\mu \mathrm{m}$ wavelength. In this simulation, the only source of loss is considered to be the mode mismatch at the SOI tip-polymer waveguide interface. The taper itself is assumed to be lossless and the reflection at the polymer-air interface is neglected. Simulations are performed for transverse-electric (TE) polarized light. Because high index contrast functional optical devices are nearly always polarization-dependent, the polarization independence of the fiber coupler is not a main concern. FIMMPROP-3D (by PhotoDesign), a commercially available fully vectorial eigenmode expansion tool, was used. The required tip width depends on the properties of both the SOI waveguide and polymer waveguide. The SOI waveguide core is assumed $220 \mathrm{~nm}$ high while the polymer waveguide 


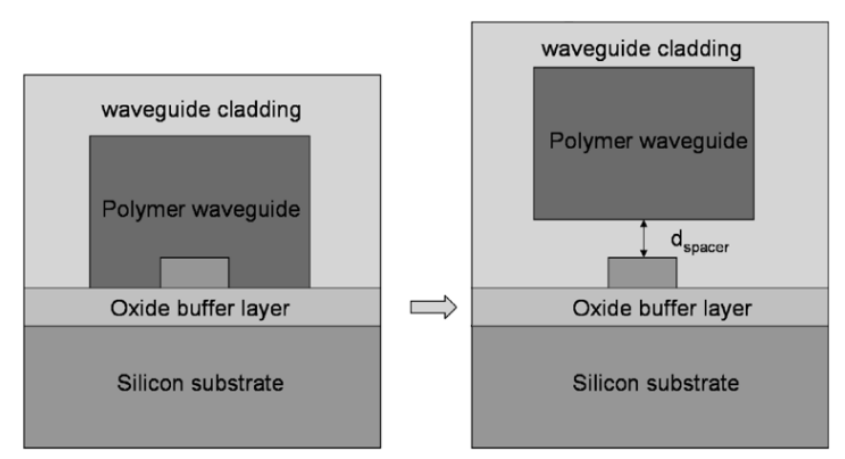

Fig. 3. Modified taper design for fabrication using a 248-nm-deep UV lithography system.

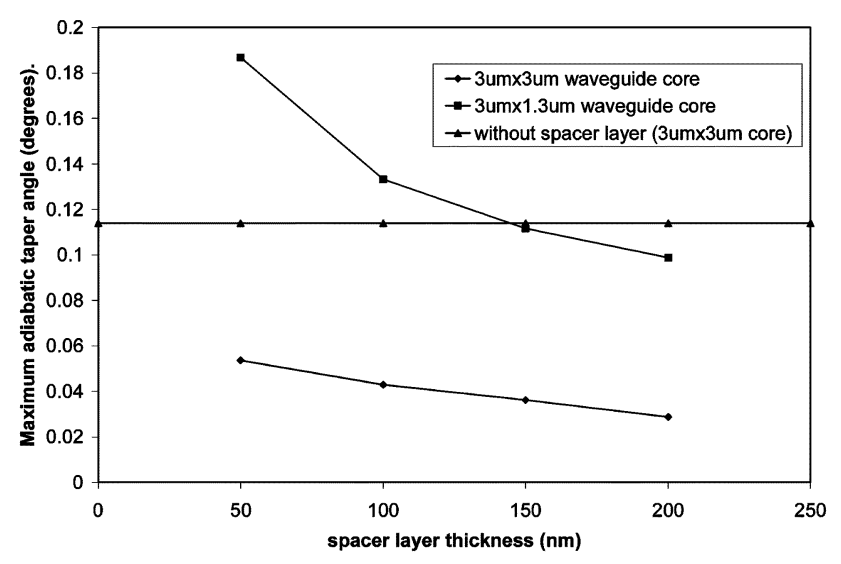

Fig. 4. Influence of the spacer thickness on the spot size converter maximum adiabatic taper angle for the modified design.

is assumed to have a refractive index of 1.67 and core dimensions of $3 \times 3 \mu \mathrm{m}^{2}$. A benzocyclobutene (BCB) top cladding $(n=1.54)$ is assumed. To assess the possibilities of a 248-nm-deep UV lithography system to fabricate the taper tips an ASML PAS5500/750 stepper was used. Using a standard resist mask process taper tips down to $175 \mathrm{~nm}$ could be fabricated. As can be seen in Fig. 2, this achievable tip width limits the efficiency of the device. To increase the efficiency, the design of the spot size converter was changed by adding a low index BCB spacer layer, as shown in Fig. 3, to reduce the coupling of the waveguide modes. The simulated efficiency of the taper device for a spacer layer of 100 and $200 \mathrm{~nm}$ is also shown in Fig. 2. This graph shows that adding this spacer layer increases the achievable efficiency using deep UV lithography to about $100 \%$. The influence of the spacer layer thickness on the maximum adiabatic taper angle (defined as the maximum taper angle to give a theoretical conversion loss of $0.1 \mathrm{~dB}$ ) of the spot size converter is shown in Fig. 4. It is clear that without altering the polymer waveguide dimensions, the spot size converter length drastically increases by adding a spacer layer. This can, however, be overcome by reducing the polymer waveguide height without reducing the efficiency for deep UV definable taper tips, as can be seen in Figs. 2 and 4, where, respectively, coupling losses and maximum taper angle for a 1.3- $\mu \mathrm{m}$ polymer core height are plotted. As is shown in Fig. 5, there is, however, a small reduction in the coupling efficiency between the polymer waveguide mode and the lensed fiber spot for reduced polymer waveguide height. It is clear that

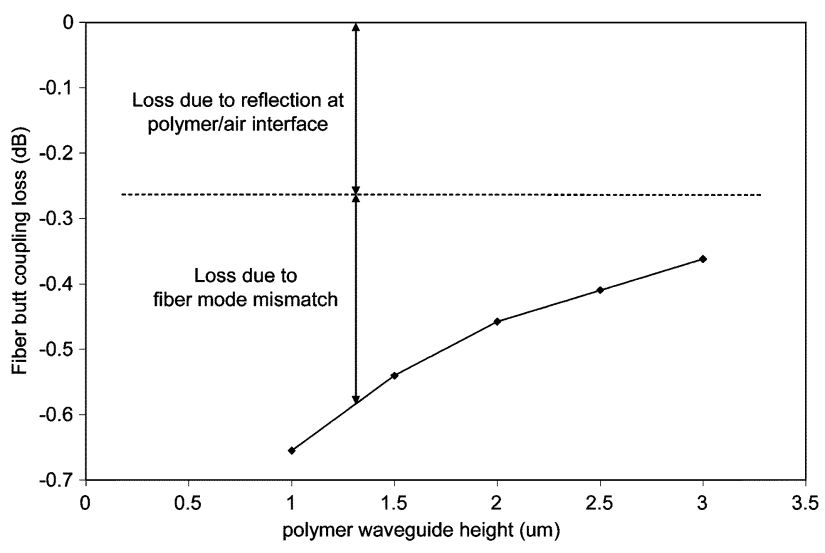

Fig. 5. Influence of the polymer waveguide height on the coupling efficiency to an optical fiber. A Gaussian lensed fiber spot of $2.5 \times 2.5 \mu \mathrm{m}^{2}$ is assumed. Simulations are performed for TE polarization at a wavelength of $1.55 \mu \mathrm{m}$.

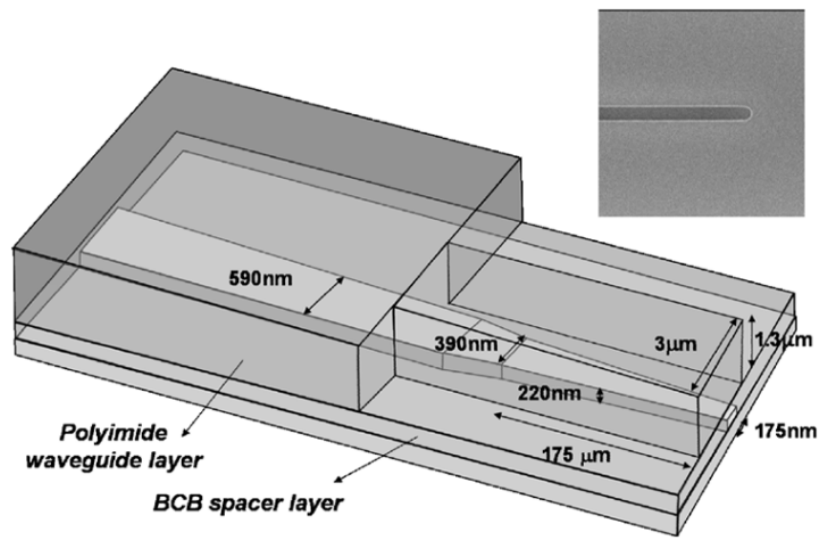

Fig. 6. SOI spot size converters: design and fabricated SOI taper tips. BCB top cladding is not shown for clarity.

the choice of polymer waveguide height is a tradeoff between device length and coupling efficiency.

\section{FABRICATION AND MEASUREMENTS}

Piecewise linear SOI spot size converters were fabricated using an ASML 248-nm PAS5500/750 stepper. The SOI waveguide dimensions are shown in Fig. 6, together with a scanning electron microscope picture of the taper tip. After waveguide fabrication, a 200-nm BCB film was spin coated on top of the SOI waveguide structure. To achieve this very thin layer thickness, BCB was diluted using mesitylene [5]. After curing, a polyimide waveguide core layer of $1.3 \mu \mathrm{m}$ thick was applied and the waveguide core was etched using a 100-nm Ti mask and ICP plasma etching. After removal of the Ti mask using diluted HF, a thick BCB topcladding was applied. The refractive index of the polyimide and $\mathrm{BCB}$ for TE polarization at $1.55 \mu \mathrm{m}$ is 1.67 and 1.54 , respectively, characterized using a Metricon 2010 prism coupling setup. The structure used to characterize the spot size converters is shown in Fig. 7. A grating coupler was used to inject light into the fundamental TE waveguide mode of the SOI waveguide, while a lensed fiber with a spot size of $2.5 \times 2.5 \mu \mathrm{m}^{2}$ or an objective lens was used to collect the light at the polymer waveguide facet. The lensed fiber had a specified loss of $0.5 \mathrm{~dB}$ while the loss 


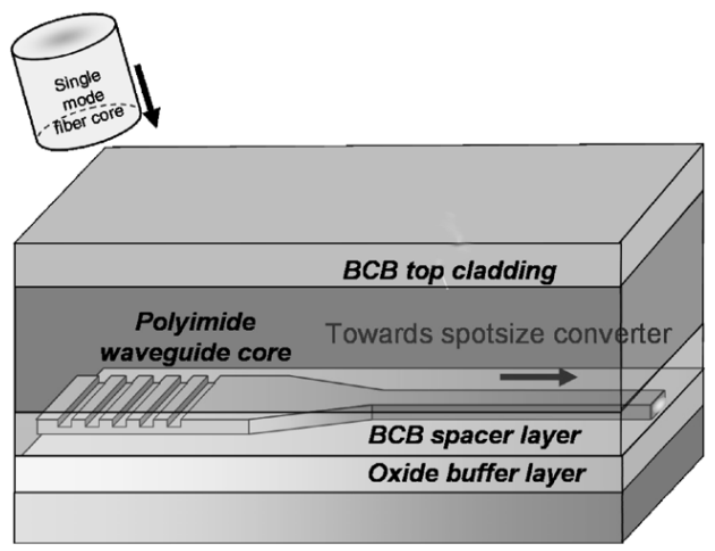

Fig. 7. Light injection using an SOI grating coupler.

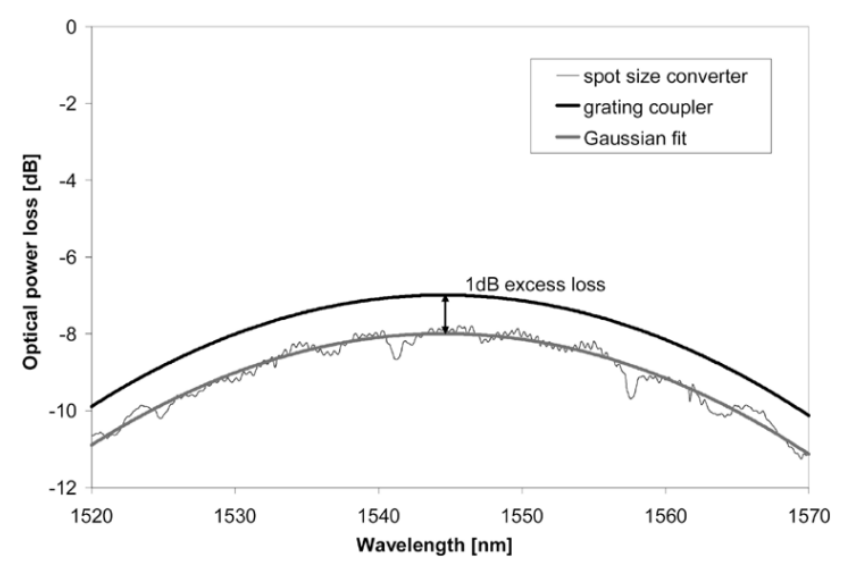

Fig. 8. Measured transmission spectrum using an objective collection lens. The grating transmission spectrum is superimposed.

of the objective collection was negligible. The grating coupler used in the experiments was characterized to have 7-dB loss and a $60-\mathrm{nm} 3-\mathrm{dB}$ bandwidth. Fig. 8 shows a transmission spectrum measured by light collection using an objective. The coupling efficiency of the grating coupler is superimposed. This implies that the SOI spot size converter itself shows 1-dB loss. Transmission experiments using a lensed fiber were also carried out. A coupling loss from lensed fiber to a 590-nm-wide SOI waveguide of $1.9 \mathrm{~dB}$ was measured. This $0.9-\mathrm{dB}$ extra loss is caused by the mode mismatch between the polymer waveguide mode and the lensed fiber mode and the additional $0.5-\mathrm{dB}$ loss of the lensed fiber.

\section{CONCLUSION}

A spot size converter for efficient coupling between an optical fiber and an SOI waveguide circuit was designed for and fabricated by 248-nm-deep UV lithography. A tradeoff has to be made between fiber chip coupling efficiency and compactness of the spotsize converter. Experimentally, 1.9-dB coupling loss was measured between a lensed fiber and an 590-nm-wide SOI waveguide.

\section{REFERENCES}

[1] W. Bogaerts, R. Baets, P. Dumon, V. Wiaux, S. Beckx, D. Taillaert, B Luyssaert, J. Van Campenhout, P. Bienstman, and D. Van Thourhout, "Nanophotonic waveguides in silicon-on-insulator fabricated with CMOS technology," J. Lightw. Technol., vol. 23, no. 1, pp. 401-412, Jan. 2005.

[2] D. Taillaert, W. Bogaerts, P. Bienstman, T. F. Krauss, P. Van Daele, I. Moerman, S. Verstuyft, K. De Mesel, and R. Baets, "An out-of-plane grating coupler for efficient butt-coupling between compact planar waveguides and single-mode fibers," J. Quantum Electron., vol. 38, no. 7, pp. 949-955, Jul. 2002.

[3] S. J. McNab, N. Moll, and Y. A. Vlasov, "Ultra-low loss photonic integrated circuit with membrane-type photonic crystal waveguides," Opt. Express, vol. 11, no. 22, pp. 2927-2937, Nov. 2003.

[4] T. Shoji, T. Tsuchizawa, T. Watanabe, K. Yamada, and H. Morita, "Spotsize converter for low-loss coupling between $0.3 \mu \mathrm{m}$ square Si wire waveguides and single mode fibers," in Proc. 15th Annu. Meeting IEEE Lasers and Electro-Optics Society, vol. 1, 2002, pp. 289-290.

[5] G. Roelkens, D. Van Thourhout, I. Christiaens, R. Baets, and M. Smit, "Ultra-thin BCB bonding for heterogeneous integration of III-V devices and SOI photonic components," in Proc. ECIO 2005, Apr. 2005, pp. $250-254$ 